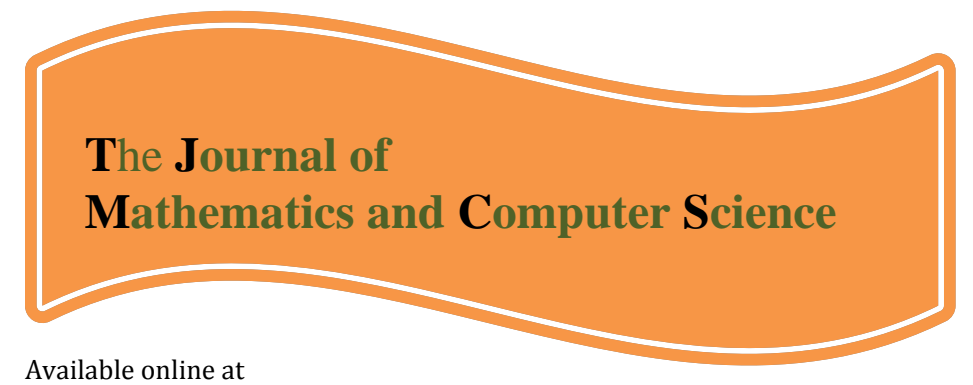

http://www.TJMCS.com

The Journal of Mathematics and Computer Science Vol .1 No.4 (2010) 287-292

\title{
Investigate of fuzzy semi-simple Lie algebra
}

\author{
A. Taleshian ${ }^{1,}$, F. Khaniani, A. A. Hosseinzadeh \\ taleshian@umz.ac.ir \\ khaniani@umz.ac.ir \\ hosseinzadeh@umz.ac.ir
}

Received: July 2010, Revised: October 2010

Online Publication: December 2010

\begin{abstract}
In this paper we have tried apply the concepts of fuzzy set to lie algebras and fuzzy ideals of lie algebras in order to provide suitable conditions to introduce the solvable fuzzy ideals, we use zadeh's extension principle the aim of this paper is to introduce and study new definition for fuzzy lie algebras and fuzzy semi-simple of lie algebras.
\end{abstract}

Keywords: Fuzzy algebra, Lie algebra, Fuzzy ideal, solvable Fuzzy ideal, Fuzzy semi-simple.

\section{Introduction}

The notion of a fuzzy subset of a nonempty set was introduced by zadeh [5]. So Rosenfeld introduced the concept of a fuzzy subgroup of a group [4]. The concept of fuzzy Lie algebra was introduced in [7].

In this paper we use the concepts of fuzzy ideals and solvable fuzzy ideals to characterize fuzzy semi simple. Relationship between the operations of Lie algebras $\varphi \times \varphi \rightarrow \varphi$ and zadeh's extension principle are studied. We also study the relationship between them and fuzzy ideals and fuzzy subalgebras. Finally solvability of fuzzy ideals are studied led to introduced fuzzy semisimple.

\section{Preliminaries}

$1,{ }^{*}$ University of Mazandaran 
A Lie algebra is a pair $(\varphi,[]$,$) where \varphi$ is a vector space over $\mathrm{F}$ and the bracket operation [,] is a F-bilinear map of $\varphi \times \varphi \rightarrow[X, Y] .[1]$

Definition 2.1A fuzzy ideal I in $\varphi$ is a fuzzy subset I which satisfiese for all $x, y \in \varphi$ and $\alpha, \beta \in F$.

$$
\begin{gathered}
I(\alpha x+\beta y) \geq I(x) \wedge I(y) \\
I([x y]) \geq I(x) \vee I(y) \\
I(0)=1 .
\end{gathered}
$$

Definition 2.2 A Fuzzy lie subalgebra or simply a fuzzy subalgebra g of $\varphi$ is a Fuzzy subset $\varphi \in \mathrm{I}^{\varphi}$ which satisfies for all $x, y \in \varphi$ and $\alpha, \beta \in F$ :

1.

2.

3.

$$
\begin{aligned}
& g(\alpha x+\beta y) \geq g(x) \wedge g(y) \\
& g([x y]) \geq g(x) \vee g(y) \\
& g(0)=1
\end{aligned}
$$

Remark : it can be show that if I is a Fuzzy ideal (or a fuzzy subalgebra) then for all $\alpha \in F$, $x \in \varphi$ we have :

$I(\alpha x)=I(x)$

The sum and multiplication operations of Zadeh's extension principle. They are defined as: $\forall A, B \in I, x \in I$

$$
\begin{aligned}
& 1-\quad(A \oplus B)(x)=\sup \{A(a) \wedge B(b) \mid a+b=x\} \\
& 2-\quad(A \cdot B)(x)=\sup \{A(a) \wedge B(b) \mid[a b]=x\}
\end{aligned}
$$

If $A \in \mathrm{I}^{\varphi}, \alpha \in F, x \in \varphi$, we define $\alpha x$ as

$$
(\alpha \cdot \mathrm{A})(\mathrm{x})=\left\{\begin{array}{lr}
A\left(\alpha^{-1} \cdot x\right) & \alpha \neq 0 \\
1 & \alpha=0, x=0 \\
0 & \alpha=0, \quad x \neq 0
\end{array}\right.
$$

Definition 2.3 A fuzzy binary relation $R$ on a set $X$ is said to be a fuzzy similarity relation, if it satisfies for all $x, y, z \in X$ :

$$
\begin{array}{ll}
1- & R(x, x)=1 \\
\text { 2- } & R(x, y)=R(y, x) \\
\text { 3- } & R(x, y) \wedge R(y, z) \leq R(x, z)
\end{array}
$$

Let $\mathrm{A}$ and $\mathrm{B}$ be sets and let $\mathrm{f}: \mathrm{A} \rightarrow \mathrm{B}$ be a function. For a fuzzy set $b$ in $B$, the inverse image of $b$ under $\mathrm{f}$ is the fuzzy set $f^{-1}(b)$ in $A$ by the rule $f^{-1}(b)(x a)=b(f(a)) \quad$ for $a \in A\left(\right.$ i.e $\left.f^{-1}(b)=b o f\right)$

Definition 2.4 Let $(\mathrm{A}, \varphi)$ and $(\mathrm{B}, \phi)$ are fts's lemma (3.2) of [9] , then a bijective mapping of $(\mathrm{A}, \varphi)$ onto $(\mathrm{B}, \phi)$ is a fuzzy homomorphism if and only if both $\mathrm{f}$ and $f^{-1}$ are fuzzy continuous.( fuzzy continuous by using (3.5) of [9]) 
Definition 2.5 Let $\varphi_{1}, \varphi_{2}$ be two Lie algebras, A Lie algebra s homomorphism $f: \varphi_{1} \rightarrow \varphi_{2}$ has a natural extension,call it $\mathrm{f}$ as well $f: I^{\varphi_{1}} \rightarrow I^{\varphi_{2}}$ defined by for all $\varphi \in I^{\varphi}, y \in \varphi_{2}$ $f(\varphi)(y)=\sup \left\{\varphi(x) \mid x \in f^{-1}(y)\right\}, f(\varphi)$ is said to the homomorphic image of the fuzzy for set $\varphi$.

Theorem 2.6 If I ,I are two fuzzy ideals in $\varphi$ then so is $I \oplus I$.

Proof: see theorem 3.3 of [2] and theorem 6.1.14 of [8].

A fuzzy similarity R on a lie algebra $\varphi$ is said to be compatible with the lie algebra operation $\mathrm{s}$ if it sastises for each $x, y, z \in \varphi, \alpha \in F$ :

1- $\quad R(x+z, y+z)=R(x, y)$

2- $\quad R([x z],[y z]) \geq R(x y)$

3- $\quad R(\alpha x, \alpha y)=R(x, y)$

A canonical one to one correspondence between fuzzy similarity relation is defined.

Let I be a fuzzy ideal in $\varphi$ for, $\forall x \in \varphi$ define the fuzzy subset $x+\mathrm{I} \in \mathrm{I}^{\varphi}$ by $(x+I)(y)=I(y-x)$ [3]. Let $I$ be an ideal in $\varphi$ for all $x, y \in \varphi$ and $\alpha \in \mathrm{F}$, then we have:

$$
\begin{gathered}
(x+I)+(y+I)=(x+y)+I \\
{[(x+I)(y+I)]=[x y]+I} \\
\alpha(x+I)=\alpha x+I
\end{gathered}
$$

For any fuzzy subset $A$ of $\varphi$ Let [A] to be the fuzzy subspace of $\varphi$ generated by A.It is intersection of all fuzzy subspaces of $\varphi$ containing $A$.

For any fuzzy subset $A$ of $\varphi$,let [A] to be the fuzzy subspace of $\varphi$,it is the instersection of all fuzzy subspaces of $\varphi$ containing A,theorem[2] and (4.2) of [8] give for all $x \in \varphi$. $[A](x)=\sup \left\{\min A\left(x_{i}\right): x=\sum \alpha_{i} x_{i}, \alpha_{i} \in F, x_{i} \in \varphi\right\}$. See theorem 7.3.2 of [8], if I and $\varphi$ are two fuzzy ideals in and $A=I,[A]$ will be denoted by $[I \varphi]$.

Lemma 2.7[8] Suppose that $A, B$ and $C$ are fuzzy ideals in $\phi$ then

$$
\begin{aligned}
& {[A \oplus B] \subseteq[A] \oplus[B] } \\
& \text { if } \quad A \subseteq B \quad \text { then } \quad[A] \subseteq[B] \\
& \\
& {[A B C]=[A[B C]] }
\end{aligned}
$$

Proof:see section (7.3) of [8], section( 4.1) of [7].

Theorem 2.8 Let I be a fuzzy ideal in $\varphi$ then [I,I] is also a fuzzy ideal in $\varphi$. 
Proof: $[\varphi[\mathrm{I}, \mathrm{I}]] \supseteq[\mathrm{I}[\mathrm{I}, \varphi]] \oplus[\mathrm{I}[\varphi, \mathrm{I}]] \subseteq[\mathrm{I}, \mathrm{I}] \oplus[\mathrm{I}, \mathrm{I}] \subseteq[\mathrm{I}, \mathrm{I}]$.

Definition 2.9 A fuzzy ideal in $\varphi$ is said to be nth derived fuzzy ideal if a sequence of fuzzy ideals in $\varphi$ by

$I^{0}=I, I^{1}=\left[I^{0}, I^{0}\right], I^{2}=\left[I^{1}, I^{1}\right], \ldots, I^{n}=\left[I^{n-1}, I^{n-1}\right], \ldots$

Then we get

$I^{0} \supseteq I^{1} \supseteq I^{2} \supseteq \ldots \supseteq I^{n} \supseteq$..

\section{Main Results}

A fuzzy ideal I in $\phi$ is called a solvable fuzzy ideal if there is a positive integer $\mathrm{n}$ such that $\eta^{n}=0$ where

$$
\eta n=\sup \left\{I^{n}(X): \varphi^{m}=x \neq 0\right\}
$$

$\eta^{n}$ is the height of $I^{n}$ but excluding 0 when taking the supremom then it is clear that $\eta 0 \geq \eta 1 \geq \eta^{2} \geq \cdots . \geq \eta n \geq \cdots$

Definition 3.1 A non-zero fuzzy Lie algebra $L$ is said to be fuzzy semi-simple if it has no non-zero solvable fuzzy ideals or equivalently if radical $L=0$.

Remark 3.2 This largest solvable fuzzy ideal is said to be the fuzzy radical of $L$ and is denoted radical $L$.

Theorem 3.3 suppose that I is an fuzzy ideal of $L$,then $L / I$ is abelian if and only if I contains the drived algebra $\boldsymbol{L}$.

Proof: the algebra $L / I$ is abelian if and only if for all $x, y \in L$, we have $[x+I, y+I]=[x, y]+I=I$ or, equivalently for all $x, y \in L$ we have $[\mathrm{x}, \mathrm{y}] \in \mathrm{I}$,since I is a fuzzy subspace of $L$, this holds iff the space spanned by brakets $[x, y]$ is contained in I; that is $L \subseteq \mathrm{L}$.

Theorem 3.4 If $L$ is a fuzzy lie algebra with fuzzy ideals $L=I_{0} \supseteq I_{1} \supseteq \ldots$ 글 $=0$ such that $\boldsymbol{I}_{\boldsymbol{k}-\mathbf{1}} / \boldsymbol{I}_{\boldsymbol{k}}$ is abelian for $1 \leq k \leq m$, then $L$ is solvable fuzzy.

Proof: We shall show that $L^{(k)}$ is contained in $I_{k}$ for $\mathrm{k}$ between 1 and m.putting $\mathrm{k}=\mathrm{m}$ will then give $L^{(m)}=0$. Since $L / I_{1}$ is abelian, we have that $L^{1} \subseteq L_{1}$, for the inductive step, we suppose that $L^{(k-1)} \subseteq I_{(k-1)}$, where $\mathrm{k} \geq 2$.the lie algebra $I_{k-1} / I_{k}$ is abelian. Therefore this time applied to the lie to the fuzzy lie algebra $I_{k-1}$, we have $\left[I_{k-1}, I_{k-1}\right] \subseteq I_{k}$. but $L^{(k-1)}$ is contained in $I_{k-1}$ by our inductive hypothesis, so we deductive hypothesis, so we deduce that 
$L^{k}=\left[L^{(k-1)}, L^{(k-1)}\right] \subseteq\left[I_{(k-1)}, I_{(k-1)}\right]$ and hence $L^{(k)} \subseteq I_{k}$.

This proof shows that if $L^{k}$ is non-zero then $I_{k}$ is also non-zero.

Theorem 3.5 Let L be a fuzzy Lie algebra:

i) if $L$ is solvable fuzzy, then every sub algebra homomorphic image of $L$ are solvable fuzzy. ii) if $I$ and $J$ are solvable fuzzy ideals of $L$, then $I+J$ is a solvable fuzzy ideal of $L$.

proof: i) If $L_{1}$ is a fuzzy subalgebra of $\mathrm{L}$,then for each $\mathrm{k}$ it is clear that $L_{1}{ }^{(k)} \subseteq L^{(k)}$, so if $L^{(m)}=0$, then also $L_{1}{ }^{(1)}=0$. For the second part, apply that $\varphi: L_{1} \rightarrow L_{2}$ is a surjective homomorphism of fuzzy lie algebras then $\varphi\left(L_{1}^{(k)}\right)=L_{2}^{(k)}$ (by using from property of being solvable fuzzy should be inherited various constructions).

ii) We have $(L / I)^{(k)}=\left(L^{(k)}+I\right) / I$. By apply to canonical homomorphism $L \rightarrow L / I$ or prove this directly by induction on $\mathrm{k}$ ) if $L / I$ is solvable fuzzy then for som $m \geq 1$ we have $(L / I)^{(m)}=0$; that is $L^{(m)}+\mathrm{I}=\mathrm{I}$ and therefore $L^{(m)} \subseteq I$.if $\mathrm{I}$ is also solvable fuzzy, then $I^{(k)}=0$.for some $\mathrm{k} \geq 1$ and hence $\left(L^{(m)}\right)^{(k)} \subseteq I^{(k)}=0$. Now one convince oneself that by definition $\left(L^{(m)}\right)^{(k)}=L^{(m+k)}$, now by using top, isomorphism theorem $(I+J) / I \cong J / I \cap J$, so it is solvable fuzzy by part (i). Since I is also solvable fuzzy and this theorem implies that $\mathrm{I}+\mathrm{J}$ is solvable fuzzy.

Theorem 3.6 Suppose that $L$ is a fuzzy Lie algebra, then the factor algebra $L /$ radical $L$ is fuzzy semi simple.

Proof: Let $\bar{J}$ be a solvable Fuzzy ideal of $L /$ radical $L$.by the fuzy ideal correspondence,there is an fuzzy ideal $\mathrm{J}$ of $\mathrm{L}$ containing rad $\mathrm{L}$ such that $\bar{J}=J / \mathrm{radL}$, by definition radL is solvable fuzzy, and $\mathrm{J} / \mathrm{radL}=\vec{\jmath}$ is solvable fuzzy by hypothesis that $\mathrm{J}$ is solvable fuzzy , but then $\mathrm{J}$ is contained in $\operatorname{rad}(L)$; that is $\bar{J}=0$.

\section{References}

[1] U. C. De and A.A.shaikh, defferential Geometry of manifolds (2007) 96-100. 
[2] A. K. Katsaras and D.B.Lin, Fuzzy vector spaces and Fuzzy topological vector spaces,j.Math.Anal.Appl. 58 (1977) 135-146.

[3] H. V. Kumbhojkar and M. S. Bapat, Correspondence theorem for fuzzy ideals fuzzy sets and systems, 41 (1991) 213-219.

[4] A. Rosenfeld, Fuzzy groups, J. Math. Anal. Appl. 35 (197l) 512-517.

[5] G. C. Muganda, fuzzy Linear and offine spaces, fuzzy sets and systems 38 (1990) 365-373.

[6] L. A. Zadeh, Fuzzy sets, Inform. and control. 8 (1965) 338-353.

[7] Samy Ei-badwy yehia, fuzzy subalgebras and fuzzy ideals of Liealgebras, fuzzy sets and systems (1996) 244-237

[8] Y. yandong, JN Mordeson and S.C.cheng, lecture notes in Fuzzy Math-ematics and computer, science Elements of Albert creighion universityomaha nebraska,usa,(1994).

[9] M .Ferraro and David H.Foster, differention of Fuzzy continuous mappings on fuzzy topological vector spaces, Mathematical Analysis and Application (1987) 589-601 